Desde las aulas

\title{
Suplementos deportivos: ¿Cómo definimos a estos productos?
}

\section{Sports supplements: How do we define these products?}

\author{
Muñoz-Maldonado, Gerardo Enrique; Ochoa-Ahmed, Fernando \\ Alberto; Díaz-Ochoa, Eduardo Alejandro; Ramírez-Orozco, Ricardo \\ Ernesto; Gómez-Renaud, Víctor Manuel
}

\author{
Gerardo Enrique Muñoz-Maldonado \\ cevam99@gmail.com \\ Universidad Autónoma de Nuevo León, México \\ Fernando Alberto Ochoa-Ahmed \\ ochoatennis@yahoo.com \\ Universidad Autónoma de Nuevo León, México \\ Eduardo Alejandro Díaz-Ochoa \\ ediazo@uanl.edu.mx \\ Universidad Autónoma de Nuevo León, México \\ Ricardo Ernesto Ramírez-Orozco \\ dcmrero@gmail.com \\ Universidad Autónoma de Aguascalientes, México \\ Víctor Manuel Gómez-Renaud \\ victor.gomezrn@uanl.edu.mx \\ Universidad Autónoma de Nuevo León, México
}

\author{
Lux Médica \\ Universidad Autónoma de Aguascalientes, México \\ ISSN: 2007-1655 \\ Periodicidad: Cuatrimestral \\ vol. 16 , núm. 48,2021 \\ luxmedica.editorial@gmail.com \\ Recepción: 25 Mayo 2021 \\ Aprobación: 15 Junio 2021 \\ URL: \\ https://revistas.uaa.mx/index.php/luxmedica/article/view/3235 \\ Autor de correspondencia: victor.gomezrn@uanl.edu.mx \\ Poítica de acceso abierto La Revista Lux Médica proporciona \\ un acceso abierto a su contenido, basado en el principio de que \\ ofrecer un acceso libre a las investigaciones ayuda a incrementar el \\ intercambio global del conocimiento. La LM no cobra ni cobrará \\ ningún cargo a sus lectores por concepto de suscripción, ni a los \\ autores por enviar, procesar o publicar sus artículos. Como condición \\ de publicación, los autores acuerdan liberar sus derechos de autor \\ bajo una licencia compartida, específicamente la licencia de Creative \\ Commons Reconocimiento-NoComercial-Compartir Igual 4.0 \\ Internacional Esta licencia permite a cualquier persona compartir, \\ copiar y redistribuir el material en cualquier medio o formato bajo \\ los siguientes términos: - Dar crédito al autor del texto - No hace
}

Resumen: Existe una gran variedad de suplementos alimenticios, los cuales pueden ser utilizados con diversas finalidades, por lo que la categorización de los suplementos deportivos ha sido compleja y hasta el día de hoy insatisfactoria en el área científica e, incluso, legal, por lo que es importante intentar contribuir a la descripción de una definición de suplemento deportivo para su contextualización en México y de esta forma homologar la terminología en futuros trabajos de investigación científica en diversas áreas.

Palabras clave: suplementos alimenticios, suplementos deportivos, ayudas ergogénicas.

Abstract: There is a wide variety of dietary supplements, which can be used for multiple purposes, whereby the categorization of sports supplements has been complex and until today unsatisfactory in the scientific and even legal area, which is why it is important to try to contribute to the description of a definition of sports supplement for its contextualization in Mexico and thus standardize the terminology in future scientific research works in various areas.

Keywords: dietary supplements, sports supplements, ergogenic aids. 
uso del material con propósitos comerciales - No transformar o modificar el material. Los autores ceden el derecho de la primera publicación a esta revista, pero conservarn sus derechos de autor, de tal forma que pueden realizar otros acuerdos contractuales

independientes y adicionales para la distribución no exclusiva de la versión del artículo publicado en esta revista ( por ejemplo, incluirlo en un repositorio institucional o publicarlo en un libro) siempre que indiquen claramente que el trabajo se publicó por primera vez en ésta.

\section{(1) (2)}

Esta obra está bajo una Licencia Creative Commons Atribución-

NoComercial-CompartirIgual 4.0 Internacional.

Cómo citar este artículo: Muñoz-Maldonado, G. E., Ochoa-Ahmed, F. A., Díaz-Ochoa, E. A., Ramírez-Orozco, R. E., Gómez Renaud5,

V. M. Suplementos deportivos: ¿Cómo definimos a estos productos? Lux Médica, 16(48). Recuperado a partir de https://revistas.uaa.mx/i ndex.php/luxmedica/article/view/3235

\section{INTRODUCCIÓN}

El consumo de suplementos alimenticios ha sido cada vez más común para la prevención, el tratamiento de deficiencias nutricionales y de enfermedades con esta etiología, así como para promover la calidad de vida y un cuerpo sano. Entre los diversos tipos de suplementos alimenticios disponibles comercialmente, se destacan los suplementos deportivos. ${ }^{1}$ Cuando nos referimos a suplementos alimenticios, no existe un consenso global sobre cómo se define a estos productos ya que diferentes términos y categorías se aplican en los diversos marcos regulatorios, a menudo en constante cambio en diferentes países y regiones. Por ejemplo, un producto considerado un suplemento alimenticio y regulado como alimento en Estados Unidos de América, pudiera ser considerado como medicina complementaria, medicamento recetado o incluso un producto potencialmente controlado en otra jurisdicción.

La situación es aún más complicada cuando se consideran países como China o India, que tienen regulaciones existentes para la medicina tradicional, o fitomedicina, la cual incluye a los botánicos crudos. ${ }^{2}$ Por otra parte, los suplementos deportivos han sido definidos y categorizados de diferentes maneras, pero, hasta la fecha, ninguna de estas es meramente satisfactoria. ${ }^{3}$ Las diferentes contextualizaciones, definiciones y categorizaciones (por ejemplo, antioxidantes, termogénicos, suplementos herbales, etc.) dificultan la determinación de los suplementos alimenticios que son consumidos regularmente por los atletas y practicantes de deportes debido a las contribuciones al rendimiento físicoatlético. ${ }^{4}$

\section{METODOLOGÍA}

El presente artículo se basó en la escala para la evaluación de la calidad de los artículos de revisión narrativa (SANRA) para guiar la síntesis de la información en una revisión narrativa de calidad. ${ }^{5}$ Revisando la literatura científica y examinando los factores relacionados con los suplementos deportivos que incluyen definiciones, términos utilizados como sinónimos y categorizaciones, la búsqueda se realizó utilizando las bases de datos PubMed, Scielo, EBSCO y 
Web of Science en un periodo de tiempo desde el 6 de julio de 2020 al 21 de mayo de 2021, sin delimitación temporal para un amplio espectro a investigar. La estrategia de la búsqueda fue utilizar descriptores "Sports supplements", "dietary supplements", "food supplements", "nutritional supplements" en combinación con "definiton", "categorization", "classification", "physical activity", "physical exercise" y "sports training" en idioma inglés y en español. Adicionalmente, se utilizaron motores de búsqueda para información relacionada a la legislación y estudios de mercado de los suplementos deportivos. Los artículos, capítulos de libros y contenidos web identificados fueron leídos en su totalidad. El escrutinio de la contextualización de la información fue realizado por tres especialistas en ayudas ergogénicas y entrenamiento con resistencias, obteniendo un total de 34 elementos los cuales fueron incluidos en este artículo.

\section{SUPLEMENTOS ALIMENTICIOS}

En nuestro país, los suplementos alimenticios son definidos de acuerdo a la fracción V del artículo 215 de la Ley General de Salud como "productos a base de hierbas, extractos vegetales, alimentos tradicionales, deshidratados o concentrados de frutas, adicionados o no, de vitaminas o minerales, que se puedan presentar en forma farmacéutica y cuya finalidad de uso sea incrementar la ingesta dietética total, complementarla o suplir algún componente”. Las formas farmacéuticas aceptadas son aquellas que se ingieren por vía oral como: cápsula, emulsión, suspensión, jarabe, polvo, soluciones y tabletas, entre otras contempladas en la Farmacopea de los Estados Unidos Mexicanos, estas no permiten formas propias de otras categorías como: confitería, parches, soluciones inyectables, entre otras. Es importante señalar que los suplementos alimenticios no son productos dirigidos para tratar, curar, prevenir o aliviar síntomas de alguna enfermedad, no sirven para el tratamiento y prevención de la obesidad o sobrepeso, no son afrodisiacos ${ }^{6}$ y en su composición no deben de tener sustancias con acción farmacológica. ${ }^{7}$ Por otra parte, la Directiva del Parlamento Europeo (2002/46/EC), utiliza el término complemento alimenticio, que es definido como un producto que intenta complementar la dieta normal, consistiendo en una fuente concentrada de un nutriente o de otras sustancias que tienen un efecto fisiológico o nutricional en forma simple o combinada, comercializados en fórmulas dosificadas, cápsulas, tabletas, píldoras $\mathrm{u}$ otras formas similares, bolsas de polvo, viales de líquido, frascos gotero y otras formas similares de líquidos y polvos, que son ingeridos en cantidades pequeñas y cuantificadas. ${ }^{8}$ Cabe señalar que el término de complemento alimenticio se encuentra en la literatura científica bajo esta misma directiva, pero se ha mencionado como suplemento alimenticio, ${ }^{9}$ nutricional, ${ }^{10,11}$ y dietético. ${ }^{12,13}$ En nuestro país, no solo entre los consumidores de estos productos sino incluso entre los profesionales médicos, de la nutrición y del deporte, se hace la diferenciación de los términos suplemento y complemento alimenticio; sin embargo, basándose en la literatura científica, no se debería considerar hacer esta diferenciación.

Por otra parte, el Congreso de Estados Unidos de América, en la Ley de Educación y Salud de Suplementos Alimenticios, aprobada en el año de 1994 (Ley Pública 104 - 417), que modificó la Enmienda de Aditivos 
Alimentarios a la Ley Federal de Alimentos, Medicamentos y Cosméticos; provee el marco regulatorio para los suplementos dietéticos y los define como productos (diferentes al tabaco) que intentan suplementar la dieta y llevan o contienen uno o más de los siguientes ingredientes dietarios: a) vitaminas; b) minerales; c) hierbas u otros botánicos; d) aminoácidos; e) sustancias dietéticas de consumo humano para suplementar la dieta incrementando el consumo total diario; o f) concentrados, metabolitos, constituyentes, extractos, o combinación de cualquier ingrediente previamente mencionado. Estos productos no deben ser presentados para su uso como alimentos convencionales o como un único elemento de una comida o de la dieta. Sus presentaciones pueden incluir cápsulas, polvos, cápsulas blandas, cápsulas de gel, tabletas, líquidos, u otras formas en las cuales estos productos puedan ser ingeridos, excluyendo los aditivos alimentarios. ${ }^{14}$ Adicionalmente, la Administración China de Alimentos y Medicamentos (Decreto 205) define a los suplementos nutricionales como productos que reponen los niveles de vitaminas y/o minerales sin proporcionar energía, y que pertenecen a la categoría de alimentos saludables. Sus funciones son complementar la insuficiencia de nutrientes, prevenir deficiencias nutricionales y reducir el riesgo de ciertas enfermedades crónicas no transmisibles. ${ }^{15}$

Las definiciones anteriormente mencionadas presentan limitaciones contextuales, ya que en una declaración en consenso por parte de miembros del Comité Olímpico Internacional (COI) se sugiere que un suplemento alimenticio es un alimento, componente alimenticio, nutriente o compuesto no alimenticio que es ingerido a propósito, adicional a la dieta habitualmente consumida, con el objetivo de conseguir un beneficio específico de salud o rendimiento. Las diversas formas en las que se presentan los suplementos alimenticios incluyen: a) alimentos funcionales, alimentos enriquecidos con nutrientes adicionales o componentes fuera de su composición nutrimental típica; b) alimentos formulados y alimentos deportivos, productos que proporcionan energía y nutrientes de una forma más conveniente que los alimentos para apoyo nutricional general (p. ej., sustitutos de comida líquidos) o para su uso dirigido al entrenamiento (p. ej., bebidas deportivas, geles, barras); c) nutrientes individuales y otros componentes de alimentos o productos herbales suministrados en forma concentrada o aislada; o d) productos multi-ingrediente que contienen varias combinaciones de los mencionados anteriormente logrando resultados similares. ${ }^{16}$ Sin embargo, si se considera el término "compuestos no alimenticios", este puede permitir la posibilidad de incluir sustancias con actividad farmacológica que mejoren el rendimiento deportivo, las cuales están prohibidas por las organizaciones rectoras del deporte. ${ }^{17}$

Los suplementos alimenticios no se deben de confundir con los alimentos complementarios, ya que estos son definidos por la Organización Mundial de la Salud (OMS) como cualquier alimento o líquido diferente a la leche materna. Esto significa que las fórmulas para infantes y fórmulas consecutivas (sustitutos de leche humana) se consideran alimentos complementarios, los cuales son requeridos durante la segunda parte del primer año de vida por razones nutricionales y de desarrollo. ${ }^{18}$ 


\section{SUPLEMENTOS DEPORTIVOS CATEGORIZACIONES

Diversos medios son comúnmente utilizados con la intención de mejorar el rendimiento deportivo y reducir el riesgo de lesiones. Las ayudas empleadas para estos objetivos, debido a sus presuntas mejoras, son consideradas como ergogénicas. ${ }^{19}$ El término ergogénico se deriva de las palabras griegas ergon (trabajo) y gennan (para producir); por lo tanto, se refiere a cualquier estrategia que mejore la capacidad de rendimiento atlético o incremente las adaptaciones fisiológicas al entrenamiento. En general, los ergogénicos deportivos nutricionales están diseñados para potenciar la producción de energía y/o mejoramiento de la composición corporal aumentando el crecimiento de la masa muscular y reduciendo la grasa corporal. ${ }^{20}$

Referente a la investigación en ciencias del deporte sobre suplementos deportivos, los criterios y protocolos (en particular los que se refieren a los deportistas de alto rendimiento) a menudo son malinterpretados por los investigadores de otras áreas, y los problemas específicos ejemplifican la diferencia entre los hallazgos generalizables que se buscan en muchas áreas de la investigación científica y las intrincadas preguntas para las que los deportistas profesionales buscan respuestas. Esto también afecta al área de la nutrición deportiva con metodologías de investigación deficientes, un bajo estándar de calidad requerido para las publicaciones y sesgo en los resultados. ${ }^{21}$

Diversos términos han sido empleados para los suplementos deportivos, tales como suplementos para mejorar el rendimiento ${ }^{22}$ y suplementos para fisicoconstructivismo, ${ }^{23}$ la cual es una disciplina deportiva estética donde la preparación para la competencia requiere un proceso intenso con entrenamiento con resistencias (comúnmente conocido como entrenamiento con pesas) para aumentar la masa muscular, seguido del intento de perder masa grasa mientras se mantiene la masa magra. ${ }^{24}$ Los jueces evalúan el físico general en cuanto al grado de proporción, simetría, tamaño muscular y calidad (densidad, separación, definición), así como el tono de la piel. ${ }^{25}$ Algunos de los suplementos para fisicoconstructivismo también se clasifican dependiendo del momento en el cual tienen que ser consumidos con respecto a la sesión de entrenamiento: los suplementos pre-entrenamiento son utilizados antes del ejercicio físico para intentar incrementar la fuerza y mantener la resistencia; otros suplementos, denominados intra-entrenamiento, son consumidos durante la sesión de ejercicio para proporcionar energía, y los suplementos post-entrenamiento promueven la recuperación del consumidor y son usados de manera posterior a la sesión de ejercicio. ${ }^{26}$

Los términos "suplementos alimenticios", "ayudas ergogénicas nutricionales", "suplementos terapéuticos nutricionales", entre otros, son utilizados para describir la gran variedad de productos que conforman colectivamente la industria de los suplementos deportivos. Así como hay una gran variedad de nombres para estos, también existen diferentes definiciones o sistemas de clasificación. ${ }^{27}$ La mayoría de los suplementos deportivos se comercializan con las afirmaciones de mejorar directamente el rendimiento físico-atlético o proporcionar un beneficio indirecto a través del apoyo para mejorar las 
adaptaciones al entrenamiento, incrementar la recuperación, mantenerse libre de enfermedades o lesiones o lograr una composición corporal óptima, ${ }^{21}$ y son recomendados con base en la evidencia de los datos que respaldan un mejor rendimiento en el ejercicio, aunque otros suplementos de manera indirecta pueden mejorar la salud, las adaptaciones al ejercicio, y la recuperación de una lesión. ${ }^{28}$

Además de la categorización de los suplementos deportivos propuesta por la Sociedad Internacional de Nutrición Deportiva (ISSN) ${ }^{17}$ y el Comité Olímpico Internacional, ${ }^{16}$ los suplementos deportivos también se categorizan de forma general con base en su uso y en la evaluación de riesgo por contener sustancias prohibidas, ${ }^{3,29}$ derivando en cinco categorías (Figura 1) agrupadas de la siguiente manera:

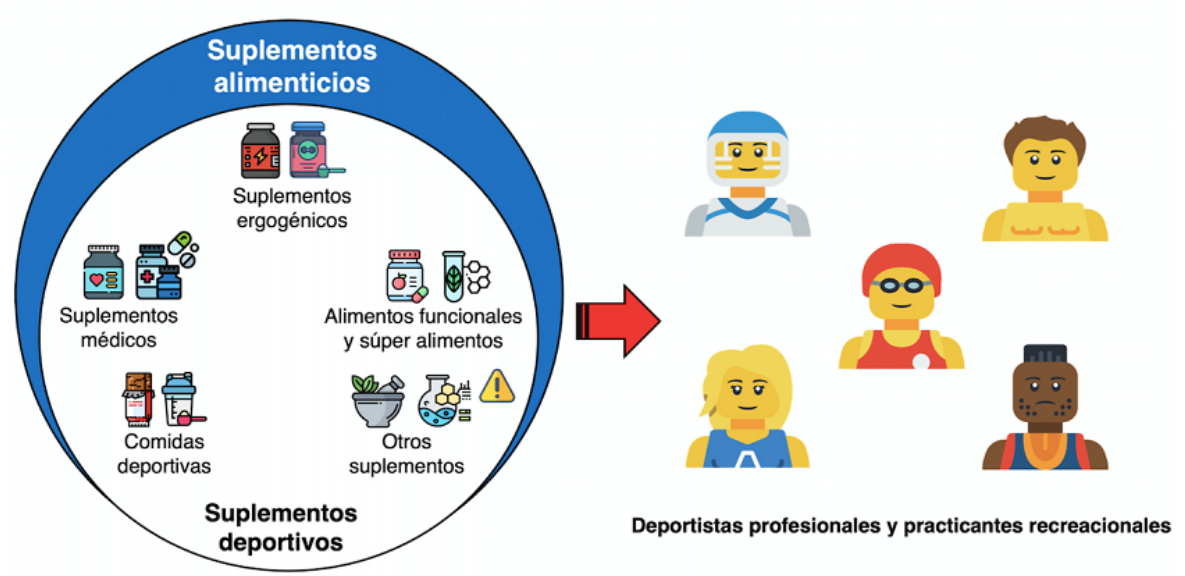

Figura 1. Categorización de los suplementos deportivos y sus consumidores

1) Comidas deportivas (bebidas deportivas, geles deportivos, comidas líquidas, confitería deportiva y barras deportivas), las cuales son productos especializados utilizados para proveer una práctica fuente de nutrientes cuando es complicado consumirlos diariamente. Estas incrementan el consumo energético por su contenido proteico y de carbohidratos.

2) Suplementos médicos (hierro, calcio, multivitamínicos/minerales, vitamina $\mathrm{D}$, probióticos, omega-3), especialmente aquellos que se utilizan para prevenir y tratar deficiencias nutricionales.

3) Suplementos ergogénicos (cafeína, creatina, b-alanina, bicarbonato de sodio y nitratos alimenticios), es decir, aquellos elaborados con ingredientes alimenticios concentrados cuyo propósito es incrementar diversas variables del rendimiento deportivo de manera específica, tales como la mejora de la capacidad de amortiguamiento y retraso de la fatiga.

4) Alimentos funcionales y súper alimentos (hierbas, algas, alimentos orgánicos, fibras vegetales, semillas, frutas alcalinizantes naturales, jugos crudos y extractos de moras), que son comidas, ingredientes alimenticios o suplementos que son ricos en compuestos considerados benéficos para la salud y el rendimiento, a menudo desconociendo sus ingredientes biológicamente activos.

5) Otros suplementos para diversas intervenciones (suplementos que afirman tener efectos de pérdida o ganancia de peso, incrementar la función inmunitaria 
e incrementar la energía, así como la masa muscular). Estos incluyen una amplia gama de extractos herbales y botánicos, a menudo en combinación con otros ingredientes, además de que algunos de estos productos tienen un riesgo elevado de contener sustancias prohibidas por la Agencia Mundial Antidopaje (WADA), tales como estimulantes del sistema nervioso central y hormonas o precursores de hormonas. Se consideran de alto riesgo de adulteración debido a la necesidad de que el consumidor obtenga resultados rápidos y notorios para promover el uso continuo del producto, y, para lograr estos resultados, los fabricantes pueden agregar sustancias con actividad farmacológica no declaradas en la etiqueta nutricional.

Otra categorización es la del Instituto Australiano del Deporte (AIS), ${ }^{30}$ el cual ha desarrollado el programa del Marco Regulatorio de los Suplementos Deportivos, diseñado para las necesidades específicas de las organizaciones deportivas australianas para promover el uso basado en la evidencia de eficacia y seguridad de algunos productos para tratar de disminuir el riesgo de dopaje inadvertido. Conocido comúnmente como el sistema de clasificación $\mathrm{ABCD}$, este incluye como subcategorías diversos grupos mencionados con anterioridad.

Aunque las diversas categorizaciones de los suplementos deportivos pueden parecer bien establecidas, el conocimiento acerca de los mismos y su aplicación práctica continúa en constante evolución. ${ }^{21}$

\section{DEFINICIONES DE SUPLEMENTOS DEPORTIVOS}

Cronológicamente, una de las primeras definiciones de suplementos deportivos fue propuesta por Maughan ${ }^{31}$ en el año de 1999, mencionándolos como ayudas ergogénicas nutricionales, las cuales tienen como objetivo mejorar el rendimiento, ya sea afectando el metabolismo energético o al sistema nervioso central, al incrementar la masa corporal magra o masa muscular por la estimulación de la síntesis proteica y reducir el contenido de grasa corporal. Posteriormente, Murray ${ }^{32}$ describe que un suplemento/producto alimenticio deportivo es cualquier alimento, bebida, tableta, gel, concentrado, polvo, cápsula, cápsula de gel o gotas líquidas que tenga el propósito de modificar la composición corporal o el estado funcional o nutricional de tal manera que sea de utilidad para las personas físicamente activas. De manera más reciente, en una revisión realizada durante 2017 por Denham ${ }^{33}$ sobre las principales fuentes de información para los deportistas en relación con la suplementación, se menciona que Eichhner y Taygart ${ }^{22}$ definen a los suplementos deportivos como productos comercializados para fisicoconstuctivismo, pérdida de peso y productos preentrenamiento/energéticos; sin embargo, al momento de revisar esta afirmación en la fuente primaria, estos autores únicamente ejemplifican a estos productos como parte de algunos suplementos alimenticios que mejoran el rendimiento deportivo. 


\section{CONSUMO DE SUPLEMENTOS DEPORTIVOS EN MÉXICO}

Es importante señalar que en un reporte de análisis de mercado realizado por el Departamento de Agricultura y Agroalimentación de Canadá (Agriculture and Agri-Food Canada) se estableció que durante el año 2008 México fue el segundo mercado más grande a nivel internacional para los suplementos deportivos con 192 millones de dólares en ventas a minoristas. ${ }^{34}$ Considerando que las ventas de suplementos deportivos, así como de herbolarios, sigue incrementándose de manera importante, ${ }^{29}$ así como la de los nutracéuticos durante la pandemia debido a sus efectos percibidos como "estimuladores del sistema inunutario" -aunque actualmente existe una escasez de evidencia científica y clínica que respalde el uso de estos productos para la prevención o mitigación contra el nuevo coronavirus SARS-CoV-2 (Síndrome respiratorio agudo severo por Coronavirus tipo 2) o la enfermedad CoV 2019 (COVID-19 [enfermedad por Coronavirus de 2019]) - ${ }^{35}$ no sería de extrañar que nuestro país siga manteniendo el segundo lugar de consumo de suplementos o ya haya superado esta posición.

\section{CONCLUSIONES}

La definición del término suplemento deportivo es importante para estudiar y discutir este grupo de productos debido al amplio grado de variabilidad de términos en la literatura científica relacionada. En conjunto, los investigadores han recopilado una gran cantidad de evidencia que respalda la contextualización de los suplementos deportivos. El desarrollo de investigaciones con datos y una terminología adecuada sobre los suplementos deportivos es de suma importancia para determinar datos más precisos y trabajos científicos de calidad en diferentes áreas relacionadas al deporte, desde nutrición hasta la salud pública. Considerando la definición más reciente de suplemento alimenticio ${ }^{16}$ y que, a su vez, no todas las disciplinas deportivas presentan los mismos requerimientos nutricionales, de rendimiento o reglamentación, ${ }^{28}$ los suplementos deportivos se pudieran llegar a definir como "fuentes e ingredientes alimenticios, así como nutrientes, que son ingeridos adicionalmente a la dieta habitual en una dosis efectiva adecuada y que pueden ser usados en ciertos periodos específicos de preparación deportiva con el propósito de incrementar el rendimiento físico, mejorando las adaptaciones al ejercicio, las habilidades, la capacidad de recuperación y corrección o la prevención de deficiencias nutricionales, así como auxiliares en la mejora de la composición corporal, ayudando a los individuos a entrenar y competir de manera más efectiva”.

\section{Agradecimientos}

La realización de la figura fue posible gracias a los elementos gráficos diseñados por dDara, Eukalyp, Freepik, Icongeek26, monkik, Photo3idea_studio, smalllikeart, Smashicons y surang disponibles en www.flaticon.com 


\section{Referencias}

1. Pereira RM, Crizel MG, La Rosa Novo D, Dos Santos CMM, Mesko MF. Multitechnique determination of metals and non-metals in sports supplements after microwave-assisted digestion using diluted acid. Microchem J. 2019; 145: 235-241. doi: 10.1016/j.microc.2018.10.043

2. Dwyer JT, Coates PM, Smith MJ. Dietary supplements: Regulatory challenges and research resources. Nutrients. 2018;10(1). doi: 10.3390/nu10010041

3. Garthe I, Maughan RJ. Athletes and supplements: Prevalence and perspectives. Int J Sport Nutr Exerc Metab. 2018; 28(2): 126-138. doi: 10.1123/ijsnem.2017-0429

4. Knapik JJ, Steelman RA, Hoedebecke SS, Austin KG, Farina EK, Lieberman HR. Prevalence of dietary supplement use by athletes: Systematic review and metaanalysis. Sport Med. 2016; 46(1): 103-123. doi: 10.1007/s40279-015-0387-7

5. Baethge C, Goldbeck-Wood S, Mertens S. SANRA - a scale for the quality assessment of narrative review articles. Res Integr Peer Rev. 2019; 4(1). doi: 10.1186/s41073-019-0064-8

6. Comisión Federal para la Protección contra Riesgos Sanitarios [Internet]. México: Gobierno de México; [actualizado en 2016 Ago 31; citado en 2021 May 28] Disponible en: https://www.gob.mx/cofepris/acciones-y-programas/ suplementos-alimenticios-62063

7. Comisión Federal para la Protección contra Riesgos Sanitarios [Internet]. México: Gobierno de México; [actualizado en 2017 Mar 03; citado en 2021 May 28] Disponible en: https://www.gob.mx/cofepris/documentos/ingredientesprohibidos-o-restringidos

8. Martínez-Sanz JM, Sospedra I, Ortiz CM, Baladía E, Gil-Izquierdo A, OrtizMoncada R. Intended or unintended doping? A review of the presence of doping substances in dietary supplements used in sports. Nutrients. 2017; 9(10): 1093. doi: $10.3390 / \mathrm{nu} 9101093$

9. Martínez-Sanz J, Sospedra I, Baladía E, Arranz L, Ortiz-Moncada R, Gil-Izquierdo A. Current Status of Legislation on Dietary Products for Sportspeople in a European Framework. Nutrients. 2017; 9(11): 1225. doi: 10.3390/nu9111225

10. Tsarouhas K, Kioukia-Fougia N, Papalexis P, et al. Use of nutritional supplements contaminated with banned doping substances by recreational adolescent athletes in Athens, Greece. Food Chem Toxicol. 2018; 115: 447-450. doi: 10.1016/ j.fct.2018.03.043

11. Savona-Ventura C, Mahmood T. EBCOG position statement about the use of herbal medication during pregnancy. Eur J Obstet Gynecol Reprod Biol. 2020; 244: 38-39. doi: 10.1016/j.ejogrb.2019.10.008

12. Lentjes MAH. The balance between food and dietary supplements in the general population. Proc Nutr Soc. 2019; 78(1): 97-109. doi: 10.1017/ S0029665118002525

13. Žeželj SP, Tomljanović A, Jovanović GK, et al. Prevalence, knowledge and attitudes concerning dietary supplements among a student population in Croatia. Int J Environ Res Public Health. 2018; 15(6): 1058. doi: 10.3390/ijerph15061058

14. Brown AC. An overview of herb and dietary supplement efficacy, safety and government regulations in the United States with suggested improvements. Part 1 of 5 series. Food Chem Toxicol. 2017; 107: 449-471. doi: 10.1016/ j.fct.2016.11.001 
15. Xia H, Liu H, Yang Y, et al. Investigation and Comparison of Nutritional Supplement Use, Knowledge, and Attitudes in Medical and Non-Medical Students in China. Nutrients. 2018; 10(11): 1810. doi: 10.3390/nu10111810

16. Maughan RJ, Burke LM, Dvorak J, et al. IOC consensus statement: Dietary supplements and the high-performance athlete. Int J Sport Nutr Exerc Metab. 2018; 28(2): 104-125. doi: 10.1123/ijsnem.2018-0020

17. Kerksick CM, Wilborn CD, Roberts MD, et al. ISSN exercise \& sports nutrition review update: Research \& recommendations. J Int Soc Sports Nutr. 2018; 15(1): 38. doi: 10.1186/s12970-018-0242-y

18. Fewtrell M. Complementary foods. In: Koletzko B, Bhatia J, Bhutta ZA, Cooper P, Makrides M, Uauy R, Wang W, editors. Pediatric Nutrition in Practice 2nd revised edition. World Review of Nutrition and Dietetics. Basel: Karger; 2015. p. 109-112.

19. Church JB, Allen TN, Allen GW. A Review of the Efficacy of Weight Training Aids. Strength Cond J. 2016; 38(3): 11-17.

20. Guimarães-Ferreira L, Cholewa JM, Silva Dantas W, Murai I, Duncan MJ, Eidy Zanchi N. Performance-Enhancing Drugs and Sports Supplements for Resistance Training. In: Bagchi D, Nair S, Sen CK, editors. Nutrition and Enhanced Sports Performance: Muscle Building, Endurance, and Strength 2nd edition. London: Academic Press; 2019. p. 31-47.

21. Burke LM. Supplements for Optimal Sports Performance. Curr Opin Physiol. 2019; 10: 156-165. doi: 10.1016/j.cophys.2019.05.009

22. Eichner A, Tygart T. Adulterated dietary supplements threaten the health and sporting career of up-and-coming young athletes. Drug Test Anal. 2016; 8(3-4): 304-306. doi: 10.1002/dta.1899

23. Jacobson IG, Horton JL, Smith B, et al. Bodybuilding, Energy, and WeightLoss Supplements Are Associated With Deployment and Physical Activity in U.S. Military Personnel. Ann Epidemiol. 2012; 22(5): 318-330. doi: 10.1016/ j.annepidem.2012.02.017

24. Kistler BM, Fitschen PJ, Ranadive SM, Fernhall B, Wilund KR. Case study: Natural bodybuilding contest preparation. Int J Sport Nutr Exerc Metab. 2014; 24(6): 694-700. doi: 10.1123/ijsnem.2014-0016

25. International Federation of Bodybuilding and Fitness: Men's Bodybuilding Rules. [Internet] 2021 [citado en 2021 May 16] Disponible en: https://ifbb.com/wpcontent/uploads/2021/03/Mens-Bodybuilding-Rules-2021.pdf

26. De Ceglie C, Calvano CD, Zambonin CG. MALDI-TOF MS for quality control of high protein content sport supplements. Food Chem. 2015; 176: 396-402. doi: 10.1016/j.foodchem.2014.12.038

27. Burke LM, Broad E, Cox G, et al. Supplements and sport foods. In: Burke LM, Deakin V, editors. Clinical Sports Nutrition 4th edition. Australia: McGraw Hill; 2010. p. 419-500.

28. Rawson ES, Miles MP, Larson-Meyer DE. Dietary supplements for health, adaptation, and recovery in athletes. Int J Sport Nutr Exerc Metab. 2018; 28(2): 188-199. doi: 10.1123/ijsnem.2017-0340

29. Garthe I. Dietary supplements and elite athletes: when nature becomes high risk. Curr Opin Endocr Metab Res. 2019; 9: 66-73. doi: 10.1016/j.coemr.2019.07.004

30. Australian Institute of Sport. Australian Institute of Sport Position Statement: Supplements and sports foods in high performance sport. [Internet] 2021 [citado en 2021 Jun 14] 
Disponible en: https://www.ais.gov.au/_data/assets/pdf_file/0014/1000841/ Position-Statement-Supplements-and-Sports-Foods-abridged_v2.pdf

31. Maughan RJ. Nutritional ergogenic aids and exercise performance. Nutr Res Rev. 1999; 12(2): 255-280. doi: 10.1079/095442299108728956

32. Murray R. Sports Nutrition Products. En Maughan RJ, editor Nutrition in Sport. Oxford: Blackwell Science Ltd; 2000. p. 524-531.

33. Denham BE. Athlete information sources about dietary supplements: A review of extant research. Int J Sport Nutr Exerc Metab. 2017; 27(4): 325-334. doi: 10.1123/ijsnem.2017-0050

34. Department of Agriculture and Agri-Food Canada. Overview of the Global Sports Nutrition Market, 10745E. [Internet]. 2010 [Citado en 2021 May 23]. Disponible en: http://publications.gc.ca/collections/collection_2015/aac-aafc/ A74-2-2010-10-eng.pdf

35. Lordan R, Rando HM, Greene CS. Dietary Supplements and Nutraceuticals under Investigation for COVID-19 Prevention and Treatment. Gilbert JA, ed. mSystems. 2021; 6(3). doi: 10.1128/msystems.00122-21 